\title{
On the invariant measure of a piecewise-smooth circle homeomorphism of Zygmund class
}

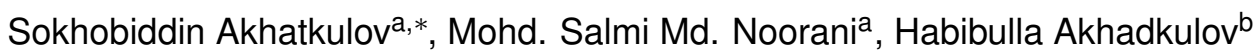 \\ a School of Mathematical Sciences, Faculty of Science and Technology, University Kebangsaan Malaysia, 43600 UKM Bangi, Selangor \\ DE, Malaysia. \\ ${ }^{b}$ School of Quantitative Sciences, University Utara Malaysia, CAS 06010, UUM Sintok, Kedah DA, Malaysia.
}

Communicated by J. Brzdek

\begin{abstract}
We prove that the invariant probability measure of an orientation preserving circle homeomorphism $f$ with several break points (at which the derivative $f^{\prime}$ has jumps) is singular with respect to Lebesgue measure, if $f^{\prime}$ satisfies certain condition and the product of jump ratios at break points is non-trivial. (C)2017 All rights reserved.
\end{abstract}

Keywords: Break point, circle homeomorphism, invariant measure, rotation number. 2010 MSC: 37E10, 37C15, $26 \mathrm{D} 99$.

\section{Introduction}

Let $S^{1}=\mathbb{R} / \mathbb{Z}$ be the unit circle with clearly defined orientation, metric, Lebesgue measure and the operation of addition. Let $\pi: \mathbb{R} \rightarrow S^{1}$ denote the corresponding projection mapping that "winds" a straight line on the circle. An arbitrary homeomorphism $f$ that preserves the orientation of the unit circle $S^{1}$ can be "lifted" on the straight line $\mathbb{R}$ in the form of the homeomorphism $F: \mathbb{R} \rightarrow \mathbb{R}$ with property $F(x+1)=F(x)+1$ that is connected with $f$ by relation $\pi \circ F=f \circ \pi$. This homeomorphism $F$ is called the lift of the homeomorphism $f$ and is defined up to an integer term. The most important arithmetic characteristic of the homeomorphism $f$ of the unit circle $S^{1}$ is the rotation number

$$
\rho(f)=\lim _{n \rightarrow \infty} \frac{F^{n}(x)}{n} \bmod 1,
$$

where $F$ is the lift of $f$ with $S^{1}$ to $\mathbb{R}$. Here and below, for a given map $F, F^{n}$ denotes its $n$-th iterate. The rotation number is rational, if and only if $f$ has periodic points. Poincare proved that, if $f$ does not have any periodic orbit, then it is semi-conjugate to the linear rotation $f_{\rho}: x \rightarrow x+\rho \bmod 1$. Denjoy [6] proved that if $f$ is a circle diffeomorphism with irrational rotation number $\rho=\rho(f)$ and $\log f^{\prime}$ is

\footnotetext{
${ }^{*}$ Corresponding author

Email addresses: akhatkulov@yahoo.com (Sokhobiddin Akhatkulov), msn@ukm.edu.my (Mohd. Salmi Md. Noorani), akhadkulov@yahoo.com (Habibulla Akhadkulov)
}

doi:10.22436/jnsa.010.01.05 
of bounded variation, then $f$ is topologically conjugate to the linear rotation $f_{\rho}$, that is, there exists an essentially unique homeomorphism $\varphi$ of the circle with $\varphi \circ f=f_{\rho} \circ \varphi$. The problem of smoothness of the conjugation $\varphi$ of smooth diffeomorphisms has come to be very well understood (see $[3,10,12-14,16])$.

It is well-known that every circle homeomorphism with irrational rotation number $\rho$ has a unique $f$ invariant probability measure $\mu_{\mathrm{f}}$. Furthermore, the conjugation $\varphi$ and the invariant probability measure $\mu_{\mathrm{f}}$ are connected by the relation $\varphi(x)=\mu_{\mathrm{f}}([0, x]), x \in S^{1}$ (see for example [4]). Because of this relation, the invariant measure $\mu_{\mathrm{f}}$ is absolutely continuous with respect to the Lebesgue measure $l$, if and only if $\varphi$ is given by an absolutely continuous function. Natural generalizations of circle diffeomorphisms are piecewise smooth homeomorphisms with break points or shortly the class of P-homeomorphisms which was introduced by Herman [10]. In general, the ergodic properties of P-homeomorphisms such as their invariant measures and renormalizations are rather different from those of diffeomorphisms (see [1, 2, 79, 15].) In [7], Dzhalilov and Khanin proved that the invariant probability measure of $C^{2+\epsilon}, \epsilon>0$ circle homeomorphism $f$ with one break point is singular with respect to Lebesgue measure. Later Dzhalilov et al. in [9] extended this result for the circle homeomorphism $f$ with several break points $b_{1}, b_{2}, \ldots, b_{n}$ such that $f^{\prime}$ is absolute continuous on $S^{1} \backslash\left\{b_{i}, i=1,2, \ldots, n\right\}, f^{\prime \prime} \in L_{1}\left(S^{1}, d l\right)$ and the jumps and their product at break points are not equal to one.

The purpose of this paper is to extend the result of Dzhalilov et al. [9]. For this, below we define a new class of circle homeomorphisms.

Let $\psi: S^{1} \rightarrow S^{1}$ be a continuous, non-decreasing function with $\psi(0)=0$. By using this function we define a class of orientation preserving circle homeomorphisms $f$ such that

$$
|f(x+t)+f(x-t)-2 f(x)| \leqslant C t \psi(t),
$$

for all $x, t \in S^{1}$ such that $x-t, x+t \in S^{1}$, here $C>0$ is a constant. The class of real functions satisfying (1.1) with $\psi \equiv 1$ on real line is called Zygmund class and denoted by $\Lambda_{*}$ (see [17]). This class plays a key role to investigate the trigonometric series. The class $\Lambda_{*}$ was applied to the circle homeomorphisms for the first time by $\mathrm{Hu}$ and Sullivan [11]. They extended the classical Denjoy's theorem to this class. The functions satisfying (1.1) are not of bounded variation at all, the reverse also is not true. For example let us consider Weierstrass function:

$$
W_{\beta}(x)=\sum_{n=1}^{\infty} \theta_{n} b^{-n \beta} \cos \left(b^{n} x\right),
$$

where $b>1$ and $\lim _{n \rightarrow \infty} \theta_{n}=0$. The following fact can be found in [17]. Weierstrass proved that for a small enough $\beta>0$ the function $W_{\beta}$ is nowhere differentiable. The extension to $\beta \leqslant 1$ was first proved by Hardy. For $\beta>1$, the function $W_{\beta}^{\prime}$ exists and continuous. If the sum of squares of the sequence $\theta_{n}$ is divergent then $W_{1}$ is differentiable in a set of measure zero. Thus by making $b$ even number and instead of $\theta_{n}$ taking the sequence $n^{-1 / 2}$, we may easily check that the function $W_{1}$ satisfies the condition (1.1), but almost nowhere differentiable and thus $W_{1}$ cannot be of bounded variation. Let $f: S^{1} \rightarrow S^{1}$ be an orientation preserving circle homeomorphism with irrational rotation number and satisfies the following conditions:

(1) There exist points $b_{1}, b_{2}, \ldots, b_{n} \in S^{1}$, the so-called break points of $f$, at which the one sided derivatives $f_{ \pm}^{\prime}\left(b_{i}\right)>0$ are defined and $\sigma\left(b_{i}\right):=\frac{f_{--}^{\prime}\left(b_{i}\right)}{f_{+}^{\prime}\left(b_{i}\right)} \neq 1, i=1, \ldots, n$;

(2) $\log f^{\prime}$ has bounded variation on $S^{1}$ and $f^{\prime}$ except break points, satisfies (1.1);

(3) $\prod_{i=1}^{n} \sigma\left(b_{i}\right) \neq 1$,

where the number $\sigma\left(b_{i}\right)$ is called jump of $f$ at the break point $b_{i}$.

Our main result is the following theorem.

Theorem 1.1. If a circle homeomorphism $f$ with irrational rotation number satisfies the above conditions (1)-(3) then its invariant probability measure $\mu_{\mathrm{f}}$ is singular with respect to Lebesgue measure $l$. 
Note that the proof of Theorem 1.1 is based on cross ratio distortion estimates and follows closely that of [9].

\section{Necessary facts and definitions}

We consider a circle homeomorphism $f$ that preserves orientation and has irrational rotation number $\rho$. Let $\left\{a_{k}, k \in \mathbb{N}\right\}$ denote the sequence of elements in the expansion of $\rho$ into a continued fraction, that is, $\rho=\left[a_{1}, a_{2}, \ldots, a_{n}, \ldots\right]$. We set $p_{n} / q_{n}=\left[a_{1}, a_{2}, \ldots, a_{n}\right], n \geqslant 1$. The numbers $p_{n} / q_{n}$ are called the convergents of $\rho$, and $q_{n}$ is the first return time. The numbers $q_{n}$ satisfy the recurrence relation

$$
\mathrm{q}_{\mathrm{n}+1}=\mathrm{a}_{\mathrm{n}+1} \mathrm{q}_{\mathrm{n}}+\mathrm{q}_{\mathrm{n}-1}, \quad \mathrm{n} \geqslant 1,
$$

with the initial conditions $q_{0}=1$ and $q_{1}=a_{1}$. For an arbitrary point $x_{0} \in S^{1}$, let $I_{0}^{(n)}\left(x_{0}\right)$ denote the closed interval with endpoints $x_{0}$ and $x_{q_{n}}=f^{q_{n}}\left(x_{0}\right)$. Note that for odd $n$ the point $x_{q_{n}}$ lies to the left of $x_{0}$, and for even $n$ to the right. We set $I_{i}^{(n)}=f^{i}\left(I_{0}^{(n)}\right), i \geqslant 1$.

Lemma 2.1 ([4]). Consider an arbitrary point $x_{0} \in S^{1}$. The segments of the trajectory $\left\{x_{i}, 0 \leqslant i<q_{n}+q_{n-1}\right\}$ divide the circle into the following disjoint (except for the endpoints) intervals:

$$
\begin{aligned}
& I_{i}^{(n)}, \quad 0 \leqslant i \leqslant q_{n-1}-1, \\
& I_{i}^{(n-1)}, \quad 0 \leqslant i \leqslant q_{n}-1 .
\end{aligned}
$$

We denote the resulting partition by $\xi_{\mathfrak{n}}\left(x_{0}\right)$ and call it a dynamical partition of order $n$. We now describe the process of transition from $\xi_{n}\left(x_{0}\right)$ to $\xi_{n+1}\left(x_{0}\right)$. All the intervals $I_{j}^{(n)}, 0 \leqslant j \leqslant q_{n-1}-1$ are preserved, and each of the intervals $I_{\mathfrak{i}}^{(n-1)}, 0 \leqslant i \leqslant q_{n}-1$ is divided into $a_{n+1}+1$ parts:

$$
I_{i}^{(n-1)}\left(x_{0}\right)=I_{i}^{(n+1)}\left(x_{0}\right) \cup \bigcup_{s=0}^{a_{n+1}-1} I_{i+q_{n-1}+s q_{n}}^{(n)}\left(x_{0}\right) .
$$

Lemma 2.2. Consider a circle homeomorphism $\mathrm{f}$ with irrational rotation number. Suppose that at points

$$
b_{i} \in S^{1}, \quad i=1,2, \ldots, k, \quad b_{1} \prec b_{2} \prec \ldots \prec b_{k},
$$

there exist positive and finite one-sided derivatives $f_{-}^{\prime}\left(b_{i}\right), f_{+}^{\prime}\left(b_{i}\right), f \in C^{1}\left(\left[b_{i}, b_{i+1}\right]\right), i=1,2, \ldots, k, b_{k+1}=b_{1}$

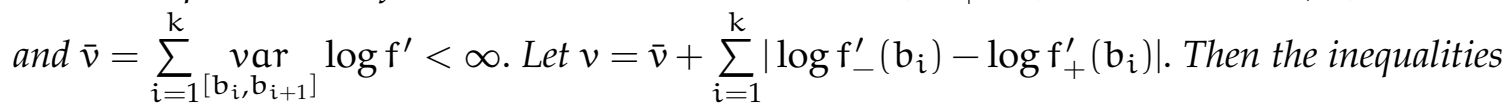

$$
e^{-v} \leqslant \prod_{s=0}^{q_{n}-1} f^{\prime}\left(f^{s}\left(y_{0}\right)\right) \leqslant e^{v}
$$

hold for any $y_{0}$ such that $\mathrm{f}^{\mathrm{s}}\left(\mathrm{y}_{0}\right) \neq \mathrm{b}_{\mathrm{i}}, \mathrm{i}=1,2, \ldots, \mathrm{k}, 0 \leqslant \mathrm{~s}<\mathrm{q}_{\mathrm{n}}$.

These inequalities are called Denjoy's inequalities. Lemma 2.2 is proved in the same fashion as the analogous assertion for diffeomorphisms (see [14]). It follows from this lemma that the intervals comprising the dynamical partition $\xi_{n}\left(x_{0}\right)$ have exponentially small lengths.

Corollary 2.3. Let $\mathrm{I}^{\mathrm{n}}$ be an arbitrary element of the dynamical partition $\xi_{n}\left(\mathrm{x}_{0}\right)$. Then

$$
l\left(I^{n}\right) \leqslant C_{1} \lambda^{n},
$$

where the constant $C_{1}$ is independent of $n, x_{0}$ and $\lambda=\left(1+e^{-v}\right)^{-1 / 2}$. 
Theorem 2.4 (Denjoy's Theorem [14]). Suppose that the hypotheses of Lemma 2.2 hold. Then the homeomorphism $f$ is topologically conjugate to the linear rotation $f_{\rho}$.

Definition 2.5 ([12]). Let $K>1$ be a constant. Two intervals $I_{1}$ and $I_{2}$ are said to be $K$-comparable on $S^{1}$, if the inequalities $\mathrm{K}^{-1} l\left(\mathrm{I}_{2}\right) \leqslant l\left(\mathrm{I}_{1}\right) \leqslant \mathrm{Kl}\left(\mathrm{I}_{2}\right)$ hold.

Definition 2.6 ([12]). An interval $I=[\tau, t] \subset S^{1}$ is said to be $q_{n}$-small, and its endpoints $q_{n}$-close, if the intervals $f^{i}(I), 0 \leqslant i \leqslant q_{n}-1$ are pairwise disjoint.

It follows from the structure of dynamical partitions that an interval $I=[\tau, t]$ is $q_{n}$-small, if and only if either $\tau<t \leqslant f^{q_{n}-1}(\tau)$ or $f^{q_{n}-1}(t) \leqslant \tau<t$.

Now we mention a notion which is called cross-ratio distortion. The cross-ratio distortion is the powerful tool to investigate the existence and smoothness of conjugation for the circle homeomorphisms with break and critical points. Note that the cross-ratio distortions were used in dynamical systems for the firs time by Yoccoz [16]. Yoccoz showed the existence of conjugation for critical circle homeomorphisms.

Definition 2.7. The cross-ratio of four real numbers $z_{1}, z_{2}, z_{3}, z_{4}$, with $z_{1}<z_{2}<z_{3}<z_{4}$, is the number

$$
\operatorname{Cr}\left(z_{1}, z_{2}, z_{3}, z_{4}\right)=\frac{\left(z_{2}-z_{1}\right)\left(z_{4}-z_{3}\right)}{\left(z_{3}-z_{1}\right)\left(z_{4}-z_{2}\right)}
$$

Definition 2.8. The cross-ratio distortion of four numbers for a strictly increasing function $F: \mathbb{R} \rightarrow \mathbb{R}$ is defined by

$$
\operatorname{Dst}\left(z_{1}, z_{2}, z_{3}, z_{4} ; \mathrm{F}\right)=\frac{\operatorname{Cr}\left(\mathrm{F}\left(z_{1}\right), \mathrm{F}\left(z_{2}\right), \mathrm{F}\left(z_{3}\right), \mathrm{F}\left(z_{4}\right)\right)}{\operatorname{Cr}\left(z_{1}, z_{2}, z_{3}, z_{4}\right)}
$$

Now we define the cross ratio distortion on the circle. Let the points $z_{1}, z_{2}, z_{3}, z_{4} \in S^{1}$, be ordered as $z_{1} \prec z_{2} \prec z_{3} \prec z_{4} \prec z_{1}$ on $S^{1}$. Let $\widehat{z}_{1}, \widehat{z}_{2}, \widehat{z}_{3}, \widehat{z}_{4}$ be the lifts of $z_{1}, z_{2}, z_{3}, z_{4}$, respectively. Note that the order of the lifts $\widehat{z}_{\mathfrak{i}}$ is not necessary to be the same as the order of points on $S^{1}$. Therefore we define $\bar{z}_{1}=\widehat{z}_{1}$ and

$$
\bar{z}_{i}= \begin{cases}\widehat{z}_{i}, & \text { if } \widehat{z}_{1}<\widehat{z}_{i}<1, \\ 1+\widehat{z}_{1}, & \text { if } 0<\widehat{z}_{1}<\widehat{z}_{i},\end{cases}
$$

where $i=2,3,4$. It is obvious that $\bar{z}_{1}<\bar{z}_{2}<\bar{z}_{3}<\bar{z}_{4}$. The vector $\left(\bar{z}_{1}, \bar{z}_{2}, \bar{z}_{3}, \bar{z}_{4}\right) \in \mathbb{R}^{4}$ is called the lifted vector of $\left(z_{1}, z_{2}, z_{3}, z_{4}\right) \in\left(S^{1}\right)^{4}$. Let $f$ be a circle homeomorphism with lift $F$. We define the cross-ratio distortion of a four-tuple $\left(z_{1}, z_{2}, z_{3}, z_{4}\right), z_{\mathfrak{i}} \in S^{1}, i=1, \ldots, 4$ ordered as $z_{1} \prec z_{2} \prec z_{4} \prec z_{1}$, with respect to $f$, by

$$
\operatorname{Dst}\left(z_{1}, z_{2}, z_{3}, z_{4} ; f\right):=\operatorname{Dst}\left(\bar{z}_{1}, \bar{z}_{2}, \bar{z}_{3}, \bar{z}_{4} ; \mathrm{F}\right) \text {. }
$$

\section{Distortion lemmas and covering intervals theorem}

In this section, we estimate the distortion of cross-ratios of four points, for the cases, when the break points of the circle homeomorphism $f$, are contained in an interval which created from the endpoints of those four points and the break points are not contained in this interval. We also provide covering intervals theorem.

Let $\omega(\delta ; f)$ denote a modulus of continuity of $f$ in the closed interval $I$, that is

$$
\omega(\delta ; f)=\left\{\sup \left|f\left(x_{1}\right)-f\left(x_{2}\right)\right| \text { for } x_{1}, x_{2} \in I,\left|x_{1}-x_{2}\right| \leqslant \delta\right\} .
$$

If $f^{\prime}$ satisfies (1.1) then $\omega\left(\delta ; f^{\prime}\right)=o\left(\delta \log \frac{1}{\delta}\right)$ (see [17]).

Lemma 3.1. Suppose that a circle homeomorphism $\mathrm{f}$ satisfies the hypotheses of Theorem 1.1. Suppose also that $z_{i} \in S^{1}, i=1, \ldots, 4$ with $z_{1} \prec z_{2} \prec z_{3} \prec z_{4} \prec z_{1}$ and the interval $\left[z_{1}, z_{4}\right]$ does not contain any break point of $\mathrm{f}$. Then

$$
\left|\operatorname{Dst}\left(z_{1}, z_{2}, z_{3}, z_{4} ; f\right)-1\right| \leqslant C_{2}\left|z_{4}-z_{1}\right| \psi\left(\left|z_{4}-z_{1}\right|\right)+\left|f^{\prime}\left(z_{4}\right)-f^{\prime}\left(z_{1}\right)\right| \omega\left(\left|z_{4}-z_{1}\right| ; f^{\prime}\right),
$$

where the constant $C_{2}$ depends only on $f$. 
Proof. We note that if $\mathrm{f}^{\prime}$ satisfies (1.1) then for each $x, y \in S^{1}$

$$
\frac{f(x)-f(y)}{x-y}=\frac{1}{x-y} \int_{x}^{y} f^{\prime}(t) d t=\frac{f^{\prime}(x)+f^{\prime}(y)}{2}+\mathcal{O}(|x-y| \psi(|x-y|)) .
$$

The proof of this equality is easy and it is proven similarly as the proof of Lemma 2.2 on page 283 in [5]. By using this equality, we get

$$
\begin{aligned}
\frac{f\left(z_{2}\right)-f\left(z_{1}\right)}{z_{2}-z_{1}} \frac{z_{4}-z_{2}}{f\left(z_{4}\right)-f\left(z_{2}\right)}= & \frac{f^{\prime}\left(z_{2}\right)+f^{\prime}\left(z_{1}\right)+\mathcal{O}\left(\left|z_{2}-z_{1}\right| \psi\left(\left|z_{2}-z_{1}\right|\right)\right)}{f^{\prime}\left(z_{4}\right)+f^{\prime}\left(z_{2}\right)+\mathcal{O}\left(\left|z_{4}-z_{2}\right| \psi\left(\left|z_{4}-z_{2}\right|\right)\right)} \\
= & \left(1-\frac{f^{\prime}\left(z_{4}\right)-f^{\prime}\left(z_{1}\right)}{f^{\prime}\left(z_{4}\right)+f^{\prime}\left(z_{2}\right)}\right)\left(1+\mathcal{O}\left(\left|z_{4}-z_{1}\right| \psi\left(\left|z_{4}-z_{1}\right|\right)\right)\right) \\
= & \left(1-\frac{f^{\prime}\left(z_{4}\right)-f^{\prime}\left(z_{1}\right)}{2 f^{\prime}\left(z_{4}\right)} \frac{1}{\left.1-\frac{f^{\prime}\left(z_{4}\right)-f^{\prime}\left(z_{2}\right)}{2 f^{\prime}\left(z_{4}\right)}\right)}\right. \\
& \times\left(1+\mathcal{O}\left(\left|z_{4}-z_{1}\right| \psi\left(\left|z_{4}-z_{1}\right|\right)\right)\right) \\
= & \left(1-\frac{f^{\prime}\left(z_{4}\right)-f^{\prime}\left(z_{1}\right)}{2 f^{\prime}\left(z_{4}\right)}\left(1+\mathcal{O}\left(f^{\prime}\left(z_{4}\right)-f^{\prime}\left(z_{2}\right)\right)\right)\right) \\
& \times\left(1+\mathcal{O}\left(\left|z_{4}-z_{1}\right| \psi\left(\left|z_{4}-z_{1}\right|\right)\right)\right) \\
= & 1-\frac{f^{\prime}\left(z_{4}\right)-f^{\prime}\left(z_{1}\right)}{2 f^{\prime}\left(z_{4}\right)}+\mathcal{O}\left(\left|z_{4}-z_{1}\right| \psi\left(\left|z_{4}-z_{1}\right|\right)\right) .
\end{aligned}
$$

In the same way can get that

$$
\frac{z_{3}-z_{1}}{f\left(z_{3}\right)-f\left(z_{1}\right)} \frac{f\left(z_{4}\right)-f\left(z_{3}\right)}{z_{4}-z_{3}}=1+\frac{f^{\prime}\left(z_{4}\right)-f^{\prime}\left(z_{1}\right)}{2 f^{\prime}\left(z_{4}\right)}+\mathcal{O}\left(\left|z_{4}-z_{1}\right| \psi\left(\left|z_{4}-z_{1}\right|\right)\right) .
$$

From (3.1) and (3.2) we obtain

$$
\begin{aligned}
\frac{f\left(z_{2}\right)-f\left(z_{1}\right)}{z_{2}-z_{1}} \frac{z_{4}-z_{2}}{f\left(z_{4}\right)-f\left(z_{2}\right)} \frac{z_{3}-z_{1}}{f\left(z_{3}\right)-f\left(z_{1}\right)} \frac{f\left(z_{4}\right)-f\left(z_{3}\right)}{z_{4}-z_{3}}= & 1-\left(\frac{f^{\prime}\left(z_{4}\right)-f^{\prime}\left(z_{1}\right)}{2 f^{\prime}\left(z_{4}\right)}\right)^{2} \\
& +\mathcal{O}\left(\left|z_{4}-z_{1}\right| \psi\left(\left|z_{4}-z_{1}\right|\right)\right) .
\end{aligned}
$$

Hence, from this equality and the modulus of continuity of $f^{\prime}$ follows that

$$
\left|\operatorname{Dst}\left(z_{1}, z_{2}, z_{3}, z_{4} ; f\right)-1\right| \leqslant \text { const }\left|z_{4}-z_{1}\right| \psi\left(\left|z_{4}-z_{1}\right|\right)+\left|f^{\prime}\left(z_{4}\right)-f^{\prime}\left(z_{1}\right)\right| \omega\left(\left|z_{4}-z_{1}\right| ; f^{\prime}\right) .
$$

The lemma is proved with const $=\mathrm{C}_{2}$.

Now we consider the case when the interval $\left[z_{1}, z_{4}\right]$ contains just one break point $b_{i_{0}}$. More precisely, suppose that $b_{i_{0}}$ lies outside the middle interval, that is, $b_{i_{0}} \in\left[z_{1}, z_{2}\right] \cup\left[z_{3}, z_{4}\right]$. Suppose for definiteness that $b_{i_{0}} \in\left[z_{1}, z_{2}\right]$. We define the numbers $\alpha, \beta, \gamma, \tau, \eta$ and $\xi$ as follows:

$$
\alpha:=z_{2}-z_{1}, \quad \beta:=z_{3}-z_{2}, \quad \gamma:=z_{4}-z_{3}, \quad \tau:=z_{2}-b_{i_{0}}, \eta:=\frac{\beta}{\alpha}, \quad \xi:=\frac{\tau}{\alpha} .
$$

Lemma 3.2. Suppose that a circle homeomorphism $\mathrm{f}$ satisfies the hypotheses of Theorem 1.1. Let $z_{\mathfrak{i}} \in \mathrm{S}^{1}, i=1, \ldots, 4$ with $z_{1} \prec z_{2} \prec z_{3} \prec z_{4} \prec z_{1}$. Suppose also that $b_{i_{0}} \in\left[z_{1}, z_{2}\right]$ and the other break points of $f$ are not contained in $\left[z_{1}, z_{4}\right]$. Then

$$
\left|\operatorname{Dst}\left(z_{1}, z_{2}, z_{3}, z_{4} ; f\right)-\frac{\left(\sigma\left(b_{i_{0}}\right)+\left(1-\sigma\left(b_{i_{0}}\right) \xi\right)\right)(1+\eta)}{\sigma\left(b_{i_{0}}\right)+\left(1-\sigma\left(b_{i_{0}}\right)\right) \xi+\eta}\right| \leqslant C_{3}\left|z_{4}-z_{1}\right| \psi\left(\left|z_{4}-z_{1}\right|\right)+\omega\left(\left|z_{4}-z_{1}\right| ; f^{\prime}\right),
$$

where the constant $\mathrm{C}_{3}>0$ depends only on $\mathrm{f}$. 
Proof. By assumption $b_{i_{0}} \in\left[z_{1}, z_{2}\right]$. Rewriting $\operatorname{Dst}\left(z_{1}, z_{2}, z_{3}, z_{4} ; f\right)$ in the form

$$
\begin{aligned}
\operatorname{Dst}\left(z_{1}, z_{2}, z_{3}, z_{4} ; f\right)=\frac{\operatorname{Cr}\left(f\left(z_{1}\right), f\left(z_{2}\right), f\left(z_{3}\right), f\left(z_{4}\right)\right)}{\operatorname{Cr}\left(z_{1}, z_{2}, z_{3}, z_{4}\right)}= & \left(\frac{f\left(z_{2}\right)-f\left(z_{1}\right)}{z_{2}-z_{1}} \cdot \frac{z_{3}-z_{1}}{f\left(z_{3}\right)-f\left(z_{1}\right)}\right) \\
& \times\left(\frac{f\left(z_{4}\right)-f\left(z_{3}\right)}{z_{4}-z_{3}} \cdot \frac{z_{4}-z_{2}}{f\left(z_{4}\right)-f\left(z_{2}\right)}\right),
\end{aligned}
$$

it is easy to check that each multiplication in parentheses equals to the following

$$
\begin{aligned}
\frac{f\left(z_{2}\right)-f\left(z_{1}\right)}{z_{2}-z_{1}} \cdot \frac{z_{3}-z_{1}}{f\left(z_{3}\right)-f\left(z_{1}\right)}= & \frac{f_{+}^{\prime}\left(b_{\mathfrak{i}_{0}}\right)\left(z_{2}-x_{\mathbf{b}}\right)+f_{-}^{\prime}\left(b_{\mathfrak{i}_{0}}\right)\left(x_{\mathbf{b}}-z_{1}\right)}{z_{2}-z_{1}} \\
& \times \frac{z_{3}-z_{1}}{f_{+}^{\prime}\left(b_{\mathfrak{i}_{0}}\right)\left(z_{3}-x_{\mathbf{b}}\right)+f_{-}^{\prime}\left(b_{\mathfrak{i}_{0}}\right)\left(x_{\mathbf{b}}-z_{1}\right)} \\
= & \frac{\left(\sigma\left(b_{\mathfrak{i}_{0}}\right)+\left(1-\sigma\left(b_{\mathfrak{i}_{0}}\right) \xi\right)(1+\eta)\right.}{\sigma\left(b_{i_{0}}\right)+\left(1-\sigma\left(b_{\mathfrak{i}_{0}}\right)\right) \xi+\eta}
\end{aligned}
$$

where $\sigma\left(b_{i_{0}}\right)=\frac{f_{-}^{\prime}\left(b_{i_{0}}\right)}{f_{+}^{\prime}\left(b_{i_{0}}\right)}$ the jump ratio of $f$ at the point $b_{i_{0}}$.

$$
\begin{gathered}
\frac{f\left(z_{4}\right)-f\left(z_{3}\right)}{z_{4}-z_{3}} \cdot \frac{z_{4}-z_{2}}{f\left(z_{4}\right)-f\left(z_{2}\right)}=\left(\frac{f^{\prime}\left(z_{4}\right)+f^{\prime}\left(z_{3}\right)}{2}+\mathcal{O}\left(\left|z_{4}-z_{3}\right| \psi\left(\left|z_{4}-z_{3}\right|\right)\right)\right) ; \\
\left(\frac{f^{\prime}\left(z_{4}\right)+f^{\prime}\left(z_{2}\right)}{2}+\mathcal{O}\left(\left|z_{4}-z_{2}\right| \psi\left(\left|z_{4}-z_{2}\right|\right)\right)\right)=\frac{f^{\prime}\left(z_{4}\right)+f^{\prime}\left(z_{3}\right)+\mathcal{O}\left(\left|z_{4}-z_{3}\right| \psi\left(\left|z_{4}-z_{3}\right|\right)\right)}{f^{\prime}\left(z_{4}\right)+f^{\prime}\left(z_{2}\right)+\mathcal{O}\left(\left|z_{4}-z_{2}\right| \psi\left(\left|z_{4}-z_{2}\right|\right)\right)}= \\
1+\frac{f^{\prime}\left(z_{3}\right)-f^{\prime}\left(z_{2}\right)}{f^{\prime}\left(z_{4}\right)+f^{\prime}\left(z_{2}\right)}+\mathcal{O}\left(\left|z_{4}-z_{1}\right| \psi\left(\left|z_{4}-z_{1}\right|\right)\right) .
\end{gathered}
$$

From (3.3) and (3.4) we have

$$
\begin{aligned}
\frac{f\left(z_{2}\right)-f\left(z_{1}\right)}{z_{2}-z_{1}} \frac{z_{3}-z_{1}}{f\left(z_{3}\right)-f\left(z_{1}\right)} \frac{f\left(z_{4}\right)-f\left(z_{3}\right)}{z_{4}-z_{3}} \frac{z_{4}-z_{2}}{f\left(z_{4}\right)-f\left(z_{2}\right)}= & \frac{\left(\sigma\left(b_{i_{0}}\right)+\left(1-\sigma\left(b_{i_{0}}\right)\right) \xi\right)(1+\eta)}{\sigma\left(b_{i_{0}}\right)+\left(1-\sigma\left(b_{i_{0}}\right)\right) \xi+\eta} \\
& +\frac{f^{\prime}\left(z_{3}\right)-f^{\prime}\left(z_{2}\right)}{f^{\prime}\left(z_{4}\right)+f^{\prime}\left(z_{2}\right)} \frac{\left(\sigma\left(b_{i_{0}}\right)+\left(1-\sigma\left(b_{i_{0}}\right)\right) \xi\right)(1+\eta)}{\sigma\left(b_{i_{0}}\right)+\left(1-\sigma\left(b_{i_{0}}\right)\right) \xi+\eta} \\
& +\mathcal{O}\left(\left|z_{4}-z_{1}\right| \psi\left(\left|z_{4}-z_{1}\right|\right)\right) .
\end{aligned}
$$

Hence, from this equality and the modulus of continuity of $f^{\prime}$ follows that

$$
\left|\operatorname{Dst}\left(z_{1}, z_{2}, z_{3}, z_{4} ; f\right)-\frac{\left(\sigma\left(b_{i_{0}}\right)+\left(1-\sigma\left(b_{i_{0}}\right) \xi\right)\right)(1+\eta)}{\sigma\left(b_{i_{0}}\right)+\left(1-\sigma\left(b_{i_{0}}\right)\right) \xi+\eta}\right| \leqslant \text { const }\left|z_{4}-z_{1}\right| \psi\left(\left|z_{4}-z_{1}\right|\right)+\omega\left(\left|z_{4}-z_{1}\right| ; f^{\prime}\right) .
$$

The lemma is proved with const $=\mathrm{C}_{3}$.

Now we introduce a concept on the intervals of the circle which cover the break points regularly. Consider $f$ with $n$ break points $b_{1}, b_{2}, \ldots, b_{n} \in S^{1}$ and irrational rotation number $\rho$. Suppose that all these break points lie in different orbits. If this were not the case, then we could achieve it by considering sufficiently high renormalizations. We set $B(f)=\left\{b_{1}, b_{2}, \ldots, b_{n}\right\}$ and say a subset $\widehat{B} \subset B(f)=\left\{b_{1}, b_{2}, \ldots, b_{n}\right\}$ is non-trivial, if $\prod_{\widehat{B}} \sigma\left(b_{i}\right) \neq 1$. We introduce the notion of a 'regular' cover of the break points in $B(f)$. Suppose that $z_{i} \in S^{1}, i=1, \ldots, 4, z_{1} \prec z_{2} \prec z_{3} \prec z_{4} \prec z_{1}$ and $r_{n}$ takes value in the set $\left\{q_{n-1}, q_{n}, q_{n-1}+q_{n}\right\}$. Suppose that the interval $\left[z_{1}, z_{4}\right]$ is $r_{n}$-small and the system of intervals $\left\{f^{j}\left(\left[z_{1}, z_{4}\right]\right), 0 \leqslant j<r_{n}-1\right\}$ covers the elements of $\widehat{B}$. We denote the number of elements of $\widehat{B}$ by $m$. For every element $b_{i_{s}} \in \widehat{B}$ there exists a number $l_{i_{s}}, 0 \leqslant l_{i_{s}}<r_{n}$ such that $\bar{b}_{i_{s}}^{(n)}=f^{-l_{i_{s}}}\left(b_{i_{s}}\right) \in\left[z_{1}, z_{4}\right]$, the point $\bar{b}_{i_{s}}^{(n)}$ is called the $r_{n}$-pre image of the element $b_{i_{s}}$ in $\left[z_{1}, z_{4}\right]$. The set of $r_{n}$-pre-images of elements of $\widehat{B}$ also consists of $m$ elements: 
$\bar{b}_{i_{1}}^{(n)}, \bar{b}_{i_{2}}^{(n)}, \ldots, \bar{b}_{i_{m}}^{(n)}$; we denote the maximal element of this set by $\widehat{b}_{t}^{(n)}$. Clearly, $\widehat{b}_{t}^{(n)}=\bar{b}_{i_{t}}^{(n)}$ for some $0 \leqslant t \leqslant m$. We introduce the following notations:

$$
\eta(j)=\frac{l\left(\left[f^{j}\left(z_{2}\right), f^{j}\left(z_{3}\right)\right]\right)}{l\left(\left[f^{j}\left(z_{1}\right), f^{j}\left(z_{2}\right)\right]\right)}, \quad \xi^{\left(i_{s}\right)}(j)=\frac{l\left(\left[f^{j}\left(\bar{b}_{i_{s}}^{(n)}\right), f^{j}\left(z_{2}\right)\right]\right)}{l\left(\left[f^{j}\left(z_{1}\right), f^{j}\left(z_{2}\right)\right]\right)}
$$

where $1 \leqslant s \leqslant m, 0 \leqslant j<r_{n}-1$. In cases, where $\bar{b}_{i_{s}}^{(n)} \in\left[z_{1}, z_{2}\right]$, the numbers $\xi^{i_{s}}(\mathfrak{j})$ are called normalized coordinates of the elements $f^{j}\left(\bar{b}_{i_{s}}^{(n)}\right)$. When the point $\bar{b}_{i_{s}}^{(n)}$ moves from $z_{2}$ to $z_{1}$, the normalized coordinate $\xi^{i_{s}}(\mathfrak{j})$ varies from 0 to 1 . It is easy to see that

$$
e^{-v} \eta(0) \leqslant \eta(j) \leqslant e^{v} \eta(0), \quad e^{-v} \xi^{i_{s}}(0) \leqslant \xi^{i_{s}}(j) \leqslant e^{v} \xi^{i_{s}}(0), \quad i=1,2, \ldots, n,
$$

for all $0 \leqslant j<r_{n}-1$ and where $v$ is the total variation of $\log f^{\prime}$ over $S^{1}$.

Definition 3.3 ([9]). Let $K>M \geqslant 1, \zeta \in(0,1), \delta>0$ be constant numbers, let $n$ be a positive integer and let $x_{0} \in S^{1}$. We say a triple of intervals $\left(\left[z_{1}, z_{2}\right],\left[z_{2}, z_{3}\right],\left[z_{3}, z_{4}\right]\right), z_{\mathfrak{i}} \in S^{1}, i=1, \ldots 4\left(K, M, \delta, \zeta, x_{0}\right)$-regularly cover the break points in a subset $\widehat{B}$, if for some $r_{n} \in\left\{q_{n-1}, q_{n}, q_{n-1}+q_{n}\right\}$ the following conditions hold:

(1) $\left[z_{1}, z_{4}\right] \subset\left(x_{0}-\delta, x_{0}+\delta\right)$ and the system of intervals $\left\{f^{j}\left(\left[z_{1}, z_{4}\right]\right), 0 \leqslant j<r_{n}-1\right\}$ covers every point in $\widehat{B}$ only once.

(2) $z_{2}=\widehat{b}_{t}^{(n)}$ and $\bar{b}_{i_{s}}^{(n)} \in\left[z_{1}, z_{2}\right), 1 \leqslant s \leqslant n, s \neq t$.

(3) $\operatorname{Ml}\left(\left[z_{2}, z_{3}\right]\right) \leqslant l\left(\left[z_{1}, z_{2}\right]\right) \leqslant \operatorname{Kl}\left(\left[z_{2}, z_{3}\right]\right)$ and $K^{-1} l\left(\left[z_{3}, z_{4}\right]\right) \leqslant l\left(\left[z_{2}, z_{3}\right]\right) \leqslant \operatorname{Kl}\left(\left[z_{3}, z_{4}\right]\right)$.

(4) The lengths of the intervals $f^{r_{n}}\left(\left[z_{1}, z_{2}\right]\right), f^{r_{n}}\left(\left[z_{2}, z_{3}\right]\right)$ and $f^{r_{n}}\left(\left[z_{3}, z_{4}\right]\right)$ are pairwise K-comparable.

(5) $\max \left\{l\left(\left[f^{r_{n}}\left(z_{i}\right), x_{0}\right]\right), l\left(\left[z_{i}, x_{0}\right]\right), i=1, \ldots, 4\right\} \leqslant K l\left(\left[z_{1}, z_{2}\right]\right)$.

(6) $\max _{1 \leqslant s \leqslant m}\left\{z^{\left(i_{s}\right)}(0)\right\}<\zeta$.

We now state a theorem on the intervals covering the break points regularly in order to use in the proof of main theorem. The proof of this theorem does not depend on the considered class of circle homeomorphisms and same with the proof of Theorem 3.1 in [9]. That is why here we provide this theorem without proof.

Theorem 3.4 ([9]). Suppose that a homeomorphism $f$ satisfies the hypotheses of Theorem 1.1. Let $x_{0} \in S^{1}$ and let $M \geqslant 1, \delta, \zeta \in(0,1)$ be constant numbers. Then there exist a constant $K=K(f, M, \zeta)>M$ such that for any sufficiently large $n$ there exists non-trivial subset $\widehat{B}=\widehat{B}(n)=\left\{b_{i_{1}}, b_{i_{2}}, \ldots, b_{i_{m}}\right\}$, points $z_{i} \in S^{1}, i=1, \ldots, 4$, $z_{1} \prec z_{2} \prec z_{3} \prec z_{4} \prec z_{1}$ and $r_{n}=r_{n}\left(z_{1}, z_{2}, z_{3}, z_{4}\right) \in\left\{q_{n-1}, q_{n}, q_{n-1}+q_{n}\right\}$ such that the intervals $\left[z_{s}, z_{s+1}\right], s=$ $1,2,3\left(K, M, \delta, \zeta, x_{0}\right)$-regularly cover the break points of $\widehat{B}$.

Note that in the proof of this theorem, the points $z_{1}, z_{2}, z_{3}, z_{4}$ were chosen and there was shown that the intervals $\left[z_{1}, z_{4}\right]$ and $\left[x_{0}, x_{q_{n-1}}\right]$ are comparable. From this follows that the intervals $\left[f^{i}\left(z_{1}\right), f^{i}\left(z_{4}\right)\right], i=$ $0,1, \ldots, r_{n}$ cover $S^{1}$ finite times. We use from this statement in the proof of Lemma 4.3.

\section{Proof of main result}

Now we recall some necessary lemmas from [9] to use in the proof of main Theorem 1.1.

Lemma 4.1 ([9]). Suppose that at a point $x=x_{0}$ the conjugation $\varphi$ has positive derivative, $\varphi^{\prime}\left(x_{0}\right)=\omega_{0}$ and the following conditions hold for some constant $\mathrm{R}_{1}>1$ :

(i) the intervals $\left[z_{1}, z_{2}\right],\left[z_{2}, z_{3}\right],\left[z_{3}, z_{4}\right]$ are pairwise $R_{1}$-comparable.

(ii) $\max \left\{\left|z_{1}-x_{0}\right|,\left|z_{4}-x_{0}\right|\right\} \leqslant R_{1}\left|z_{1}-z_{2}\right|$. 
In this case, for any $\epsilon>0$ there exists $\delta=\delta\left(x_{0}, \epsilon\right)>0$ such that if all $z_{i}, 1 \leqslant i \leqslant 4$ belong to $\left(x_{0}-\delta, x_{0}+\delta\right)$, the inequality

$$
\left|\operatorname{Dist}\left(z_{1}, z_{2}, z_{3}, z_{4} ; \varphi\right)-1\right|<\mathrm{C}_{4} \epsilon
$$

holds, where the constant $\mathrm{C}_{4}=\mathrm{C}_{4}\left(\mathrm{R}_{1}, \mathrm{w}_{0}\right)$ depends on $\mathrm{R}_{1}, \mathrm{w}_{0}$ and does not depend on $\epsilon$.

Before recalling the next lemma we define the functions $F_{i}(x, y), i=1,2, \ldots, n$ on the domain $\{(x, y)$ : $x>0,0 \leqslant y \leqslant 1\}$ as:

$$
F_{i}(x, y)=\frac{\left[\sigma\left(b_{i}\right)+\left(1-\sigma\left(b_{i}\right)\right) y\right](1+x)}{\sigma\left(b_{i}\right)+\left(1-\sigma\left(b_{i}\right)\right) y+x}
$$

where $\sigma\left(b_{i}\right)$ is the jump of $f$ at the point $b_{i}$.

Lemma 4.2 ([9]). Let $\left\{b_{i_{1}}, b_{i_{2}}, \ldots, b_{i_{m}}\right\}$ be an arbitrary non-trivial subset of break points of $f$, so that $A:=$ $\prod_{s=1}^{m} \sigma\left(b_{i_{s}}\right) \neq 1$. Then there exist constants $\Omega_{0}=\Omega_{0}\left(\sigma_{i_{1}}, \sigma_{i_{2}}, \ldots, \sigma_{i_{m}}\right)>1$ and $\tau_{0}=\tau_{0}\left(\sigma_{i_{1}}, \sigma_{i_{2}}, \ldots, \sigma_{i_{m}}\right) \in(0,1)$ such that the inequality

$$
\left|\prod_{s=1}^{m} F_{i_{s}}\left(x_{s}, y_{s}\right)-A\right| \leqslant \frac{|A-1|}{8}
$$

holds for all $x_{s} \geqslant \Omega_{0}, y_{s} \in\left[0, \tau_{0}\right], s=1,2, \ldots, m$.

We use $\tau_{0}$ and $\Omega_{0}$ to define two new constants $\overline{\tau_{0}}$ and $\overline{\Omega_{0}}$, which will play an important role in the proof of Theorem 1.1. We set $\overline{\tau_{0}}=\min \tau_{0}\left(\sigma_{i_{1}}, \sigma_{i_{2}}, \ldots, \sigma_{i_{m}}\right) \in(0,1), \overline{\Omega_{0}}=\max \Omega_{0}\left(\sigma_{i_{1}}, \sigma_{i_{2}}, \ldots, \sigma_{i_{m}}\right)$ where the minimum and maximum are taken over all non-trivial subsets $\left\{b_{i_{1}}, b_{i_{2}}, \ldots, b_{i_{m}}\right\}$ of break points of $f$.

Proof of Theorem 1.1. Suppose that a homeomorphism $f$ satisfies the hypotheses of Theorem 1.1. Since the rotation number $\rho$ is irrational, the invariant measure $\mu_{f}$ has no atoms and the conjugation $\varphi(x)$ is given by monotonic function $\mu_{\mathrm{f}}([0, x]), x \in \mathrm{S}^{1}$. The finite derivative $\varphi^{\prime}(x)$ of the conjugation exists by the monotonicity of the function $\varphi(x)$ for almost all $x$ with respect to Lebesgue measure. We claim that $\varphi^{\prime}(x)=0$ at all points $x$ where the finite derivative exists. Suppose that $\varphi^{\prime}\left(x_{0}\right)=\omega_{0}>0$ at some point $x_{0} \in S^{1}$. We fix $\epsilon>0$. Let $\delta=\delta\left(x_{0}, \epsilon\right)>0$ be defined by Lemma 4.1. We use the constants $\overline{\Omega_{0}}$ and $\overline{\tau_{0}}$ to define new constants: $M_{0}=\overline{\Omega_{0}} e^{v}, \zeta_{0}=\overline{\tau_{0}} e^{v}$ where $v>0$ is the total variation of $\log f^{\prime}$ over $S^{1}$. Let $K_{0}=K_{0}\left(f, M_{0}, \zeta_{0}\right)>M_{0}>1$ be the constant defined Theorem 3.4. By that theorem, for sufficiently large $n$, there exist a non-trivial subset $\widehat{B}=\left\{b_{i_{1}}, b_{i_{2}}, \ldots, b_{i_{m}}\right\}$ of break points of $f$, points $z_{i} \in S^{1}, i=1, \ldots, 4$ with $z_{1} \prec z_{2} \prec z_{3} \prec z_{4} \prec z_{1}$ and a number $r_{n} \in\left\{q_{n-1}, q_{n}, q_{n-1}+q_{n}\right\}$ such that the triple of intervals $\left(\left[z_{1}, z_{2}\right],\left[z_{2}, z_{3}\right],\left[z_{3}, z_{4}\right]\right)\left(K_{0}, M_{0}, \delta, \zeta_{0}, x_{0}\right)$-regularly cover the points of $\widehat{B}$. Since after $r_{n}$ steps the images of the triple of intervals $\left(\left[z_{1}, z_{2}\right],\left[z_{2}, z_{3}\right],\left[z_{3}, z_{4}\right]\right)$ cover all points of the non-trivial subset $\widehat{B}$, the cross-ratio $\operatorname{Cr}\left(z_{1}, z_{2}, z_{3}, z_{4}\right)$ and $\operatorname{Cr}\left(f^{r_{n}}\left(z_{1}\right), f^{r_{n}}\left(z_{2}\right), f^{r_{n}}\left(z_{3}\right), f^{r_{n}}\left(z_{4}\right)\right)$ are substantially different.

More precisely, the following lemma holds.

Lemma 4.3. The inequality

$$
\left|\operatorname{Dst}\left(z_{1}, z_{2}, z_{3}, z_{4} ; f^{r_{n}}\right)-1\right| \geqslant R_{2}
$$

holds for sufficiently large $n$, where the constant $R_{2}>0$ depends only on $\mathrm{f}$.

We will give the proof of this lemma later. Since the intervals $\left[z_{s}, z_{s+1}\right], s=1,2,3\left(K_{0}, M_{0}, \delta, \zeta_{0}, x_{0}\right)$ regularly cover the points in $\widehat{B}$ these intervals along with $\left[f^{r_{n}}\left(z_{s}\right), f^{r_{n}}\left(z_{s+1}\right)\right], s=1,2,3$ satisfy conditions (i), (ii) of Lemma 4.1 with constant $R_{1}=K_{0}$. By using the assertion of Lemma 4.1 we obtain

$$
\left|\operatorname{Dst}\left(z_{1}, z_{2}, z_{3}, z_{4} ; \varphi\right)-1\right| \leqslant \mathrm{C}_{4} \epsilon,
$$

$$
\left|\operatorname{Dst}\left(f^{r_{n}}\left(z_{1}\right), f^{r_{n}}\left(z_{2}\right), f^{r_{n}}\left(z_{3}\right), f^{r_{n}}\left(z_{4}\right) ; \varphi\right)-1\right| \leqslant C_{4} \epsilon
$$


where the constant $C_{4}>0$ depends on $R_{1}$ and $\omega$.

Since $\varphi$ effects a conjugation to a linear rotation, it is easy to see that

$$
\operatorname{Cr}\left(\varphi\left(f^{r_{n}}\left(z_{1}\right)\right), \varphi\left(f^{r_{n}}\left(z_{2}\right)\right), \varphi\left(f^{r_{n}}\left(z_{3}\right)\right), \varphi\left(f^{r_{n}}\left(z_{4}\right)\right)\right)=\operatorname{Cr}\left(\varphi\left(z_{1}\right), \varphi\left(z_{2}\right), \varphi\left(z_{3}\right), \varphi\left(z_{4}\right)\right) .
$$

Equations (4.2), (4.3), (4.4) immediately imply that

$$
\left|\operatorname{Dst}\left(z_{1}, z_{2}, z_{3}, z_{4} ; \mathrm{f}^{r_{n}}\right)-1\right| \leqslant C_{5} \epsilon,
$$

where the constant $C_{5}>0$ is independent of $\epsilon$ and $n$. The relations (4.1) and (4.5) cannot hold simultaneously for sufficiently small $\epsilon$. This contradiction proves Theorem 1.1.

Proof of Lemma 4.3. Recall that the triple of intervals $\left(\left[z_{1}, z_{2}\right],\left[z_{2}, z_{3}\right],\left[z_{3}, z_{4}\right]\right),\left(K_{0}, M_{0}, \delta, \zeta_{0}, x_{0}\right)$-regularly cover a non-trivial subset $\widehat{B}=\left\{b_{i_{1}}, b_{i_{2}}, \ldots, b_{i_{m}}\right\}$ of break points. By Definition 3.3 we have $z_{2}=\bar{b}_{i_{t}}^{(n)}$ and $\bar{b}_{i_{s}}^{(n)} \in\left[z_{1}, z_{2}\right), s=1,2, \ldots m, s \neq t$. We rewrite $\operatorname{Dst}\left(z_{1}, z_{2}, z_{3}, z_{4} ; f^{r_{n}}\right)$ in the form

$$
\begin{aligned}
& \operatorname{Dst}\left(z_{1}, z_{2}, z_{3}, z_{4} ; f^{r_{n}}\right)=\prod_{s=1}^{m} \operatorname{Dst}\left(f^{l_{i_{s}}}\left(z_{1}\right), f^{l_{i_{s}}}\left(z_{2}\right), f^{l_{i_{s}}}\left(z_{3}\right), f^{l_{i_{s}}}\left(z_{4}\right) ; f\right) \\
& \times \prod_{\substack{p=0 \\
p \neq l_{i_{s}}, s=1, \ldots, m}}^{r_{n}-1} \operatorname{Dst}\left(f^{p}\left(z_{1}\right), f^{p}\left(z_{2}\right), f^{p}\left(z_{3}\right), f^{p}\left(z_{4}\right) ; f\right) .
\end{aligned}
$$

Now we estimate the first factor in (4.6). The assertion of Lemma 3.2 and the definition of $F_{i}(x, y)$ imply that

$$
\begin{aligned}
\operatorname{Dst}\left(f^{l_{i_{t}}}\left(z_{1}\right), f^{l_{i_{t}}}\left(z_{2}\right), f^{l_{i_{t}}}\left(z_{3}\right), f^{l_{i_{t}}}\left(z_{4}\right) ; f\right) & =\frac{\sigma\left(\bar{b}_{i_{s}}^{(n)}\right)\left(1+\eta\left(l_{i_{t}}\right)\right)}{\sigma\left(\bar{b}_{i_{s}}^{(n)}\right)+\eta\left(l_{i_{t}}\right)}+\theta_{t}\left(z_{1}, z_{4}\right) \\
& =F_{i_{t}}\left(\eta\left(l_{i_{t}}\right), 0\right)+\theta_{t}\left(z_{1}, z_{4}\right),
\end{aligned}
$$

where

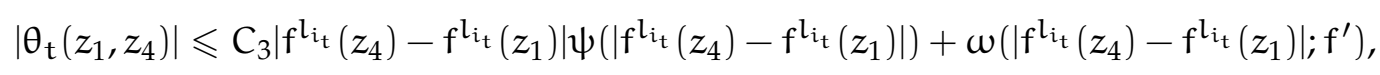

and

$$
\begin{aligned}
\operatorname{Dst}\left(f^{l_{i_{s}}}\left(z_{1}\right), f^{l_{i_{s}}}\left(z_{2}\right), f^{l_{i_{s}}}\left(z_{3}\right), f^{l_{i_{s}}}\left(z_{4}\right) ; f\right) & =\frac{\left(\sigma\left(\bar{b}_{i_{s}}^{(n)}\right)+\left(1-\sigma\left(\bar{b}_{i_{s}}^{(n)}\right)\right) \xi^{i_{s}}\left(l_{i_{s}}\right)\right)\left(1+\eta\left(l_{i_{s}}\right)\right)}{\sigma\left(\bar{b}_{i_{s}}^{(n)}\right)+\left(1-\sigma\left(\bar{b}_{i_{s}}^{(n)}\right)\right) \xi^{i_{s}}\left(l_{i_{s}}\right)+\eta\left(l_{i_{s}}\right)}+\theta_{s}\left(z_{1}, z_{4}\right) \\
& =F_{i_{t}}\left(\eta\left(l_{i_{s}}\right), \xi^{i_{s}}\left(l_{i_{s}}\right)\right)+\theta_{s}\left(z_{1}, z_{4}\right), \quad s=1,2, \ldots, m, \quad s \neq t,
\end{aligned}
$$

where also

$$
\left|\theta_{s}\left(z_{1}, z_{4}\right)\right| \leqslant C_{3}\left|f^{l_{i_{s}}}\left(z_{4}\right)-f^{l_{i_{s}}}\left(z_{1}\right)\right| \psi\left(\left|f^{l_{i_{s}}}\left(z_{4}\right)-f^{l_{i_{s}}}\left(z_{1}\right)\right|+\omega\left(\left|f^{l_{i_{s}}}\left(z_{4}\right)-f^{l_{i_{s}}}\left(z_{1}\right)\right| ; f^{\prime}\right),\right.
$$

$\mathrm{s}=1,2, \ldots, \quad \mathrm{m}, \mathrm{s} \neq \mathrm{t}$.

By construction, the interval $\left[z_{1}, z_{4}\right]$ is $r_{n}$-small and therefore the intervals $\left[f^{j}\left(z_{1}\right), f^{j}\left(z_{4}\right)\right], 0 \leqslant j<r_{n}-1$ are pairwise disjoint (except for endpoints). Hence, by using assertion of Corollary 2.3 we have

$$
\left|\mathrm{f}^{\mathrm{j}}\left(z_{4}\right)-\mathrm{f}^{\mathrm{j}}\left(z_{1}\right)\right| \leqslant \mathrm{C}_{1} \lambda^{\mathrm{n}}, \quad 0 \leqslant j<\mathrm{r}_{\mathrm{n}}-1,
$$

where $C_{1}$ is a constant and $\lambda=\left(1+e^{-v}\right)^{-1 / 2}$. Because of the properties of modulus of continuity of $f$, that is

- $\omega(\delta ; f)$ is non-decreasing function of $\delta$;

$-\omega(a \delta ; f) \leqslant([a]+1) \omega(\delta ; f)$ for any $a>0$ real number, where [.] is an integer part of number, 
and (4.9) we have

$$
\omega\left(\left|f^{l_{i s}}\left(z_{4}\right)-f^{l_{i s}}\left(z_{1}\right)\right| ; f^{\prime}\right) \leqslant\left(\left[C_{1}\right]+1\right) \omega\left(\lambda^{n} ; f^{\prime}\right),
$$

for all $0 \leqslant l_{i_{s}}<r_{n}-1$. In particular, this inequality holds for any $s=1,2, \ldots, m$. Similarly, by monotonicity of $\psi$ we have also

$$
\psi\left(\left|f^{l_{i}}\left(z_{4}\right)-f^{l_{i_{s}}}\left(z_{1}\right)\right|\right) \leqslant \psi\left(C_{1} \lambda^{n}\right),
$$

for all $0 \leqslant l_{i_{s}}<r_{n}-1$ and particularly for any $s=1,2, \ldots, m$. So if we fix $\epsilon$, then there exists $N=N(\epsilon) \geqslant 1$ such that the estimate

$$
\left|\theta_{s}\left(z_{1}, z_{4}\right)\right| \leqslant C_{1} \lambda^{n} \psi\left(C_{1} \lambda^{n}\right)+\left(\left[C_{1}\right]+1\right) \omega\left(\lambda^{n} ; f^{\prime}\right) \leqslant \epsilon, \quad s=1,2, \ldots, m,
$$

holds for all $n \geqslant N$. Suppose that $\eta(0)$ and $\xi^{i_{s}}(0), s=1,2, \ldots, m$ satisfy the following relations $\eta(0)>$ $\overline{\Omega_{0}} e^{v}=M_{0}$ and $\xi^{i_{s}}(0)<\overline{\tau_{0}} e^{-v}, s=1,2, \ldots, m$. Hence by using the relation (3.5) we obtain that $\eta\left(l_{i_{s}}\right)>\overline{\Omega_{0}}$ and $\xi^{i_{s}}\left(l_{i_{s}}\right)<\overline{\tau_{0}}, s=1,2, \ldots, m$. It follows from Lemma 4.2 that

$$
\left|F_{i_{t}}\left(\eta\left(l_{i_{t}}\right), 0\right) \prod_{s=1, s \neq t}^{m} F_{i_{t}}\left(\eta\left(l_{i_{s}}\right), \xi^{i_{s}}\left(l_{i_{s}}\right)\right)-A\right| \leqslant \frac{|A-1|}{8} .
$$

By combining (4.7)-(4.13), for sufficiently small $\epsilon>0$ we obtain

$$
\left|\left(F_{i_{t}}\left(\eta\left(l_{i_{t}}\right), 0\right)+\theta_{t}\right) \prod_{s=1, s \neq t}^{m}\left(F_{i_{t}}\left(\eta\left(l_{i_{s}}\right), \xi^{i_{s}}\left(l_{i_{s}}\right)\right)+\theta_{s}\right)-A\right| \leqslant \frac{|A-1|}{4} .
$$

Now we estimate second factor in (4.6). Since $\left[z_{1}, z_{4}\right]$ is $r_{n}$-small, we have

$$
\sum_{j=0}^{r_{n}-1}\left|f^{j}\left(z_{4}\right)-f^{j}\left(z_{1}\right)\right| \leqslant 1
$$

We can write the second factor in (4.6) in the following form

$$
\begin{aligned}
& \left|\prod_{\substack{p=0 \\
p \neq l_{i_{s}}, s=1, . ., m}} \operatorname{Dst}\left(f^{p}\left(z_{1}\right), f^{p}\left(z_{2}\right), f^{p}\left(z_{3}\right), f^{p}\left(z_{4}\right) ; f\right)-1\right| \\
& =\left|\exp \left\{\sum_{\substack{p=0 \\
p \neq l_{i_{s}}, s=1, . ., m}}^{r_{n}-1} \log \left[1+\left(\operatorname{Dst}\left(f^{p}\left(z_{1}\right), f^{p}\left(z_{2}\right), f^{p}\left(z_{3}\right), f^{p}\left(z_{4}\right) ; f\right)-1\right)\right]\right\}-1\right| \\
& \leqslant\left|\exp \left\{\sum_{\substack{p=0 \\
p \neq l_{i_{s}}, s=1, . ., m}}^{r_{n}-1} \operatorname{Dst}\left(f^{p}\left(z_{1}\right), f^{p}\left(z_{2}\right), f^{p}\left(z_{3}\right), f^{p}\left(z_{4}\right) ; f\right)-1\right\}-1\right| .
\end{aligned}
$$

By using Lemma 3.1 and inequalities (4.10), (4.11) we obtain

$$
\begin{aligned}
& \sum_{\substack{p=0 \\
p \neq l_{i_{s}}, s=1, \ldots, m}}^{r_{n}-1}\left|\operatorname{Dst}\left(f^{p}\left(z_{1}\right), f^{p}\left(z_{2}\right), f^{p}\left(z_{3}\right), f^{p}\left(z_{4}\right) ; f\right)-1\right| \\
& \leqslant \sum_{\substack{p=0 \\
p \neq l_{i_{s}}, s=1, . ., m}}^{r_{n}-1} C_{2}\left|f^{p}\left(z_{4}\right)-f^{p}\left(z_{1}\right)\right| \psi\left(\left|f^{p}\left(z_{4}\right)-f^{p}\left(z_{1}\right)\right|\right)
\end{aligned}
$$




$$
\begin{aligned}
& +\sum_{\substack{p=0 \\
p \neq l_{i_{s}}, s=1, ., m}}^{r_{n}-1}\left|f^{\prime}\left(f^{p}\left(z_{4}\right)\right)-f^{\prime}\left(f^{p}\left(z_{1}\right)\right)\right| \omega\left(\left|f^{p}\left(z_{4}\right)-f^{p}\left(z_{1}\right)\right| ; f^{\prime}\right) \\
& \leqslant \psi\left(C_{1} \lambda^{n}\right) \sum_{\substack{p=0 \\
p \neq l_{i_{s}}, s=1, . ., m}}^{r_{n}-1}\left|f^{p}\left(z_{4}\right)-f^{p}\left(z_{1}\right)\right| \\
& +\left(\left[C_{1}\right]+1\right) \omega\left(\lambda^{n} ; f^{\prime}\right) \sum_{\substack{p=0 \\
p \neq l_{i_{s}}, s=1, . ., m}}^{r_{n}-1}\left|f^{\prime}\left(f^{p}\left(z_{4}\right)\right)-f^{\prime}\left(f^{p}\left(z_{1}\right)\right)\right| .
\end{aligned}
$$

Because the intervals $\left[f^{p}\left(z_{1}\right), f^{p}\left(z_{4}\right)\right], p=0,1, \ldots, r_{n}-1, p \neq l_{i_{s}}, s=1,2, \ldots, m$ cover $S^{1}$ finite times, rewriting them as a non-overlapping intervals and using (4.15), we obtain

$$
\sum_{\substack{p=0 \\ p \neq l_{i_{s}}, s=1, \ldots, m}}^{r_{n}-1}\left|\operatorname{Dst}\left(f^{p}\left(z_{1}\right), f^{p}\left(z_{2}\right), f^{p}\left(z_{3}\right), f^{p}\left(z_{4}\right) ; f\right)-1\right| \leqslant \psi\left(C_{1} \lambda^{n}\right)+K\left(\left[C_{1}\right]+1\right) \underset{S^{1}}{\operatorname{varf}} f^{\prime} \cdot \omega\left(\lambda^{n} ; f^{\prime}\right),
$$

where $K$ is the number of covers. Hence, for sufficiently large $n$, the right side of (4.17) is less than $\epsilon$ and from this it follows that the right side of (4.16) is less than $\epsilon$, that is,

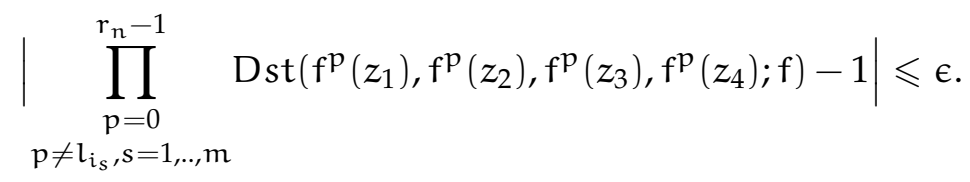

This inequality with sufficiently small $\epsilon$ and (4.14) implies that

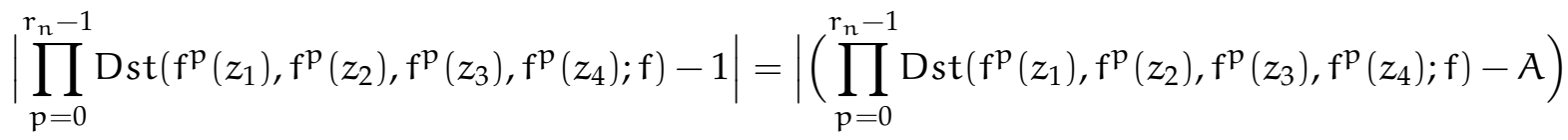

$$
\begin{aligned}
& +(A-1) \mid \\
& \geqslant \| \prod_{p=0}^{r_{n}-1} \operatorname{Dst}\left(f^{p}\left(z_{1}\right), f^{p}\left(z_{2}\right), f^{p}\left(z_{3}\right), f^{p}\left(z_{4}\right) ; f\right)-A|-| A-1|| \\
& \geqslant \frac{3|A-1|}{4} \text {. }
\end{aligned}
$$

Hence, from this inequality follows the assertion of the lemma with constant $R_{2}=\frac{3|A-1|}{4}$. Lemma 4.3 is proved.

\section{Acknowledgment}

The paper is supported via the grants DIP-2014-034, DPP-2015-FST, FRGS/1/2014/ST06/UKM/01/1 and FRGS/S/O13558.

\section{References}

[1] Kh. A. Akhadkulov, On some circle homeomorphisms with break-type singularities, (Russian) Uspekhi Mat. Nauk, 61 (2006), 183-184, translation in Russian Math. Surveys, 61 (2006), 981-983. 1

[2] H. Akhadkulov, M. S. M. Noorani, S. Akhatkulov, Renormalizations of circle diffeomorphisms with a break-type singularity, ArXiv, 2015 (2015), 26 pages. 1 
[3] V. I. Arnol'd, Small denominators, I, Mapping the circle onto itself, (Russian) Izv. Akad. Nauk SSSR Ser. Mat., 25 (1961), 21-68. 1

[4] I. P. Cornfeld, S. V. Fomin, Ya. G. Sinaĭ, Ergodic theory, Translated from the Russian by A. B. Sosinskiŭ, Grundlehren der Mathematischen Wissenschaften [Fundamental Principles of Mathematical Sciences], Springer-Verlag, New York, (1982). 1, 2.1

[5] W. de Melo, S. van Strien. One-dimensional dynamics, Ergebnisse der Mathematik und ihrer Grenzgebiete (3) [Results in Mathematics and Related Areas (3)], Springer-Verlag, Berlin, (1993). 3

[6] A. Denjoy, Sur les courbes définies par les équations différentielles á la surface du tore, J. Math. Pure Appl., 11 (1932), 333-376. 1

[7] A. A. Dzhalilov, K. M. Khanin, On an invariant measure for homeomorphisms of a circle with a point of break, Funct. Anal. Appl., 32 (1998), 153-161. 1

[8] A. Dzhalilov, I. Liousse, D. Mayer, Singular measures of piecewise smooth circle homeomorphisms with two break points, Discrete Contin. Dyn. Syst., 24 (2009), 381-403.

[9] A. A. Dzhalilov, D. Maîer, U. A. Safarov, Piecewise-smooth circle homeomorphisms with several break points, (Russian) Izv. Ross. Akad. Nauk Ser. Mat., 76 (2012), 101-120, translation in Izv. Math., 76 (2012), 94-112. 1, 1, 3.3, 3, 3.4, 4, $4.1,4.2$

[10] M. R. Herman, Sur la conjugaison différentiable des difféomorphismes du cercle á des rotations, (French) Inst. Hautes tudes Sci. Publ. Math., 49 (1979), 5-233. 1

[11] J. Hu, D. P. Sullivan, Topological conjugacy of circle diffeomorphisms, Ergodic Theory Dynam. Systems, 17 (1997), 173-186. 1

[12] Y. Katznelson, D. Ornstein, The absolute continuity of the conjugation of certain diffeomorphisms of the circle, Ergodic Theory Dynam. Systems, 9 (1989), 681-690. 1, 2.5, 2.6

[13] J. Moser, A rapidly convergent iteration method and non-linear differential equations, II, Ann. Scuola Norm. Sup. Pisa, 20 (1966), 499-535.

[14] Ya. G. Sină̆, K. M. Khanin, Smoothness of conjugacies of diffeomorphisms of the circle with rotations, (Russian) Uspekhi Mat. Nauk, 44 (1989), 57-82, translation in Russian Math. Surveys, 44 (1989), 69-99. 1, $2,2.4$

[15] E. B. Vul, K. M. Khanin, Homeomorphisms of the circle with fracture-type singularities, (Russian) Uspekhi Mat. Nauk, 45 (1990), 189-190, translation in Russian Math. Surveys, 45 (1990), 229-230. 1

[16] J. C. Yoccoz, Il n'y a pas de contre-exemple de Denjoy analytique, (French) [There are no analytic Denjoy counterexamples] C. R. Acad. Sci. Paris Sér. I Math., 298 (1984), 141-144. 1, 2

[17] A. Zygmund, Trigonometric series, Vol. I, II, Third edition, With a foreword by Robert A. Fefferman, Cambridge Mathematical Library, Cambridge University Press, Cambridge, (2002). 1, 3 